\title{
SINGLE-CHIP CMOS ANEMOMETER
}

\author{
F. Mayer, A. Häberli, H. Jacobs, G. Ofner, O. Paul, and H. Baltes
}

Physical Electronics Laboratory

ETH Zurich, CH-8093 Zurich, Switzerland

\begin{abstract}
For the first time a packaged single-chip anemometry microsystem is reported. The system includes a thermal CMOS flow sensor with on-chip power management, signal conditioning, and A/D conversion. It is fabricated using an industrial IC process followed by post-CMOS micromachining. The system is packaged on a flexible substrate using flip-chip interconnection technology. The measurement of wind speeds is demonstrated in the range from 0 to $38 \mathrm{~m} / \mathrm{s}$ ( 0 to 12 Beaufort), with a dynamic range of $65 \mathrm{~dB}$. Total power consumption is $3 \mathrm{~mW}$.
\end{abstract}

\section{Introduction}

Most miniaturized thermal flow sensors fabricated using IC technology [1-6] require off-chip driving and signal conditioning components. An early flow sensor microsystem with integrated interface circuitry was based on IC technology with additional process steps allowing subsequent micromachining [7]. Thermoelectric flow sensors fabricated using commercial CMOS processes were recently reported [8-12]. Their fabrication exploits CMOS compatible silicon micromachining [13] developed for a wide range of thermoelectric microtransducers. The cointegration of a CMOS flow sensor with an on-chip sigma/delta converter was based on this technology [14]. However, the device was encapsulated in a laboratory-type package and was unsuited for applications in real environments. This paper reports the first packaged CMOS anemometer microsystem featuring a thermoelectric flow sensor with on-chip power management, signal conditioning, and $\mathrm{A} / \mathrm{D}$ conversion. The encapsulation of the system entailed three challenges. First the sensor has to be in contact with the surrounding air while being protected against mechanical contact and undesired media. Second, to ensure long-term operation, the circuitry has to be sealed from the environment. Last but not least, the packaging solution should guarantee high reliability at low cost. With integrated circuits, the latter two requirements are now routinely fulfilled using flip-chip technology [15]. In this paper we report an elegant extension of the flip-chip method, and thereby successfully address the first challenge.

\section{Sensor Design and Fabrication}

The device was fabricated using the commercial $2 \mu \mathrm{m}$ double-poly double-metal CMOS process of Microelectronic Marin SA (EM), Switzerland, followed by anisotropic silicon etching [16]. The flow sensor is schematically shown in Fig. 1. It is based on a membrane composed of the entire sandwich of dielectric layers of the CMOS process. Along a symmetry line, the membrane contains a $6 \mu \mathrm{m}$ wide integrated gate polysilicon line with a resistance of $1.9 \mathrm{k} \Omega$, used as a heater. Temperature increases $\Delta T_{A}=T_{A}-T_{0}$ and $\Delta T_{B}=T_{B}-T_{0}$ of the membrane with respect to the die temperature $T_{0}$ are mea-

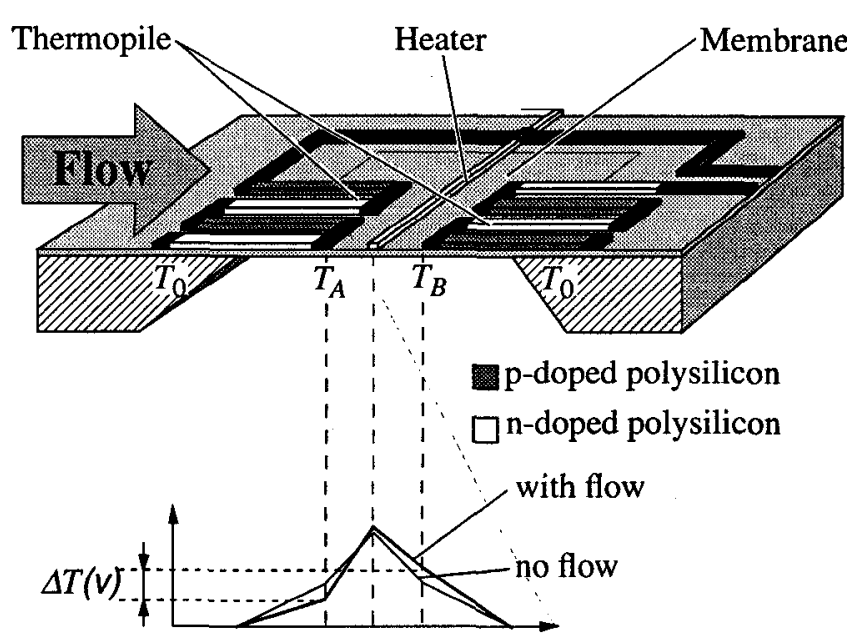

Fig. 1. Schematic view and operating principle of the micromachined thermal air flow sensor fabricated using CMOS technology. For clarity, only the lowest CMOS dielectric is shown.

sured at two locations with integrated thermopiles. Each thermopile consists of twenty bidoped gate-polysilicon/capacitorpolysilicon thermocouples. Their hot contacts are located at a distance of $130 \mu \mathrm{m}$ from the membrane edge. The cold contacts are placed above the silicon substrate. Respective thermopile output voltages $U_{A}=20 \alpha \Delta T_{A}$ and $U_{B}=20 \alpha \Delta T_{B}$ are obtained, where $\alpha=0.415 \mathrm{mV} / \mathrm{K}$ denotes the relative Seebeck coefficient of the $\mathrm{n}$-doped gate polysilicon against the p-doped capacitor polysilicon. The voltage difference $U_{\text {Sens }}=U_{B}-U_{A}$ is a flow velocity dependent signal: Its polarity gives the flow direction. The output $U_{\text {Sens }}$ typically ranges from -40 to $+40 \mathrm{mV}$, with offset below $0.5 \mathrm{mV}$ in still air. The internal resistance of each thermopile is $850 \mathrm{k} \Omega$. In order to optimize the sensor structures, the dependence of the device output characteristics on design parameters were previously determined by experimental and modeled by finite element analysis [10].

\section{On-Chip Circuitry}

A block diagram of the driving and readout electronics is shown in Fig. 2(a). The power management drives the heating resistor of the flow sensor. Depending on the wind velocity to be measured, the overall amplification $A$ of the two-stage amplifier can be set to 25 or 250 for wind velocity $v$ ranging from 0 to $0.5 \mathrm{~m} / \mathrm{s}$ and from 0.5 to $40 \mathrm{~m} / \mathrm{s}$, respectively. The amplified output is then converted into a bitstream by an incremental A/D converter [17], see Fig. 2(b). In order to minimize the pin count, decimation of the bitstream is performed off-chip by a counter. The amplifiers and A/D converter are implemented in fully differential switched capacitor technique. Fig. 3 shows the microsystem chip. 
(a)

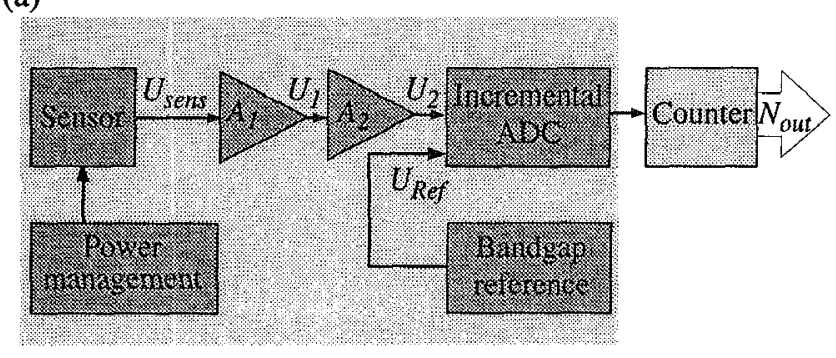

(b)

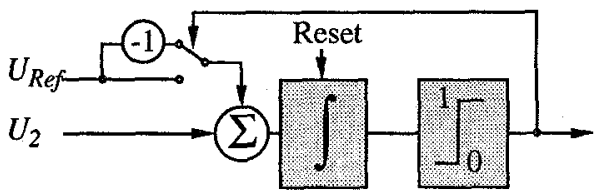

Fig. 2. (a) Microsystem block diagram with sensor (output $U_{\text {sens }}$ ), amplifiers $A_{1}$ and $A_{2}$, incremental $A / D$ converter, and power management; $(b)$ block diagram of the incremental $A / D$ converter, with input $U_{2}$ and reference $U_{R e f}$.

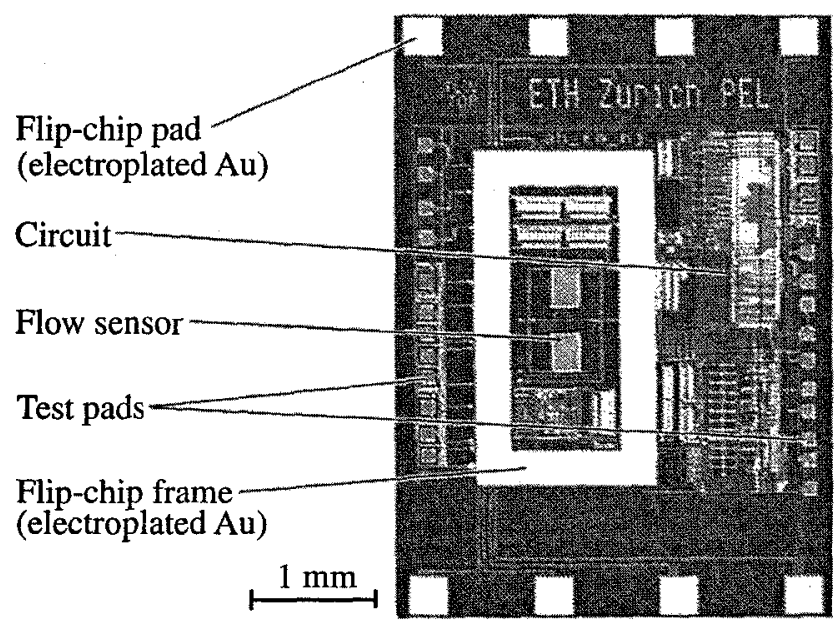

Fig. 3. Photograph of chip with integrated flow sensor and on-chip circuit including two amplifier stages, $A / D$ converter, and power management. The fip-chip frame is used to seal the sensor from the circuit.

\section{Microsystem Packaging}

The microsystem die is flip-chip [15] mounted on a flexible substrate (flex) fabricated by Dyconex AG, Switzerland, as shown in Fig. 4. The flex is plugged into a standard socket, as shown in Fig. 5. An opening in the flex substrate is aligned with the sensor and enables free advection. For the flip-chip assembly, $25 \mu \mathrm{m}$ high Au bumps and a Au frame surrounding the sensor were electroplated onto the chip using the standard bumping process of EM. The electrical interconnections between the chip bumps and the substrate contact pads were soldered with $\mathrm{PbIn}_{50}$. In the same fabrication step the bump frame was soldered to a corresponding structure on the substrate, thus sealing the electronics from the medium flowing over the sensor membrane. Finally an underfill is applied between chip and substrate. Figs. 4(a) and (b) show the front and rear of the chip/flex unit. (a)

(b)
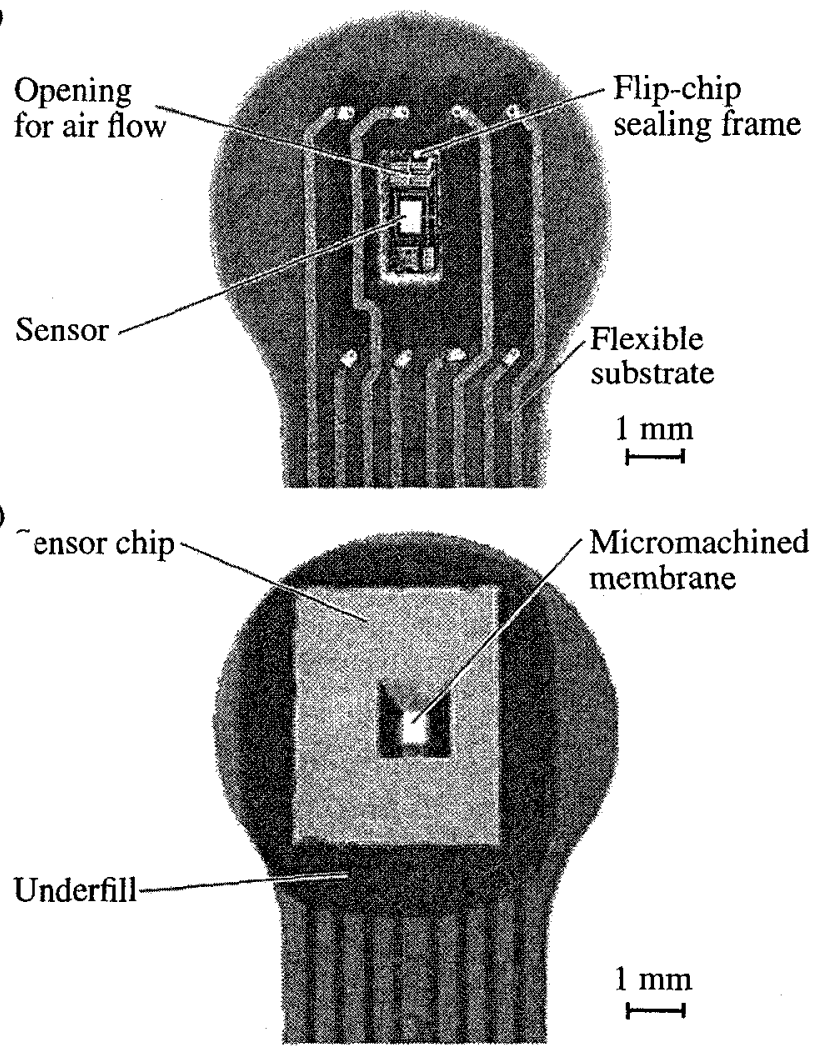

Fig. 4. (a) Front and (b) rear view of microsystem die fip-chip attached on flexible substrate.

The assembly is then inserted into a plastic housing consisting of a cylinder with a $1.0 \mathrm{~mm}$ wide flow channel, as schematically shown in Fig. 6 . The sensor is tangent to the channel. Figs. 5(a) and (b) show the device before insertion into the plastic housing. Fig. 5(c) shows the finished anemometer.

\section{Experimental}

In view of its large dynamic range, the anemometer was characterized using two different setups. First, for the velocity range from $V=0.02 \mathrm{~m} / \mathrm{s}$ to $0.5 \mathrm{~m} / \mathrm{s}$ the anemometer was moved trough still air using a stepping motor. The sensor velocity was controlled with an accuracy of $0.005 \mathrm{~m} / \mathrm{s}$. Second, for air velocities above $0.5 \mathrm{~m} / \mathrm{s}$, the anemometer was characterized in a laminar flow wind-tunnel covering flow velocities up to $38 \mathrm{~m} / \mathrm{s}$ (12 Beaufort). Azimuth $\Phi$ and inclination $\Theta$ of the anemometer with respect to the air flow direction were varied by computer controlled DC motors. At the sensor location the wind-tunnel was $25 \mathrm{~cm}$ high and $80 \mathrm{~cm}$ wide. The air velocity was measured using a propeller anemometer (MiniAir ${ }^{\circledR}$ ) of Schildknecht, Switzerland, with an accuracy better than $1.5 \%$ of the full scale output in the range from 1 to $38 \mathrm{~m} / \mathrm{s}$.

\section{Results}

Figs. 7(a) and (b) show the digital anemometer output as a function of wind speed. The anemometer was mounted in upright position. For the results in Figs. 7(a) and (b), the over- 


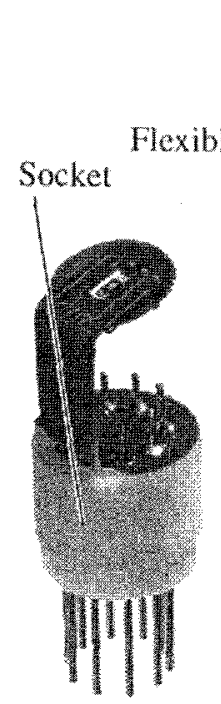

(a)

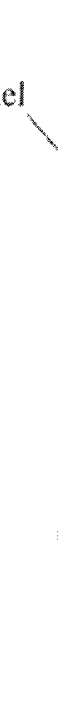

(b)

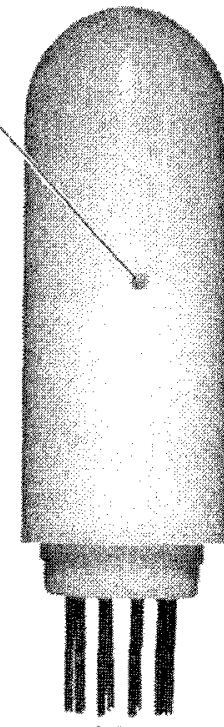

(c)

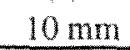

Fig. 5. Chip/flex/socket unit $(a, b)$ before and (c) after insertion into plastic housing.

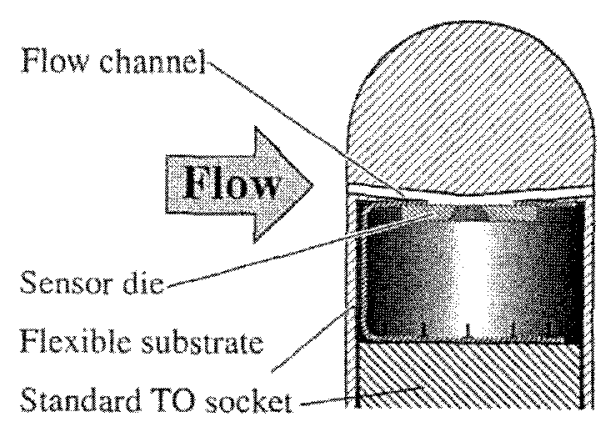

Fig. 6. Schematic cross-section of anemometer. The flip-chip mounted sensor chip is tangent to the flow channel.

all amplification $A$ was set to 25 and 250 respectively. The integration time of the $A / D$ converter was set to 0.19 and $0.75 \mathrm{~s}$, respectively, providing resolutions of 12 and 14 bit. Wind measurements are demonstrated between 0.02 and $38 \mathrm{~m} / \mathrm{s}$, which corresponds to a dynamic range of $65 \mathrm{~dB}$. The system output is nonlinear and levels off at high wind speeds. Conversion of the raw output signal into wind velocity can be performed, e.g., using a look-up table.

The dependence of the anemometer response on its orientation with respect to the wind direction is shown in Fig. 8. The output variation with the azimuth $\Phi$ is reported in Fig. 8(a) for a device in upright position. At angles close to $0^{\circ}$ and $180^{\circ}$ (channel parallel to flow) the output follows $\cos (\Phi)$. At azimuthal angles $\Phi$ between $60^{\circ}$ and $120^{\circ}$, the anemometer output shows two secondary lobes. These are caused by the combination of the Bernoulli effect [18] and boundary layer delamination. They correlate with the pressure distribution caused by the flow around a circular cylinder [19]. Fig. 8(b) shows similar results for a sensor inclined by $\Theta=15^{\circ}$ towards or $\Theta=-15^{\circ}$ away from the wind direction. For the whole (a)

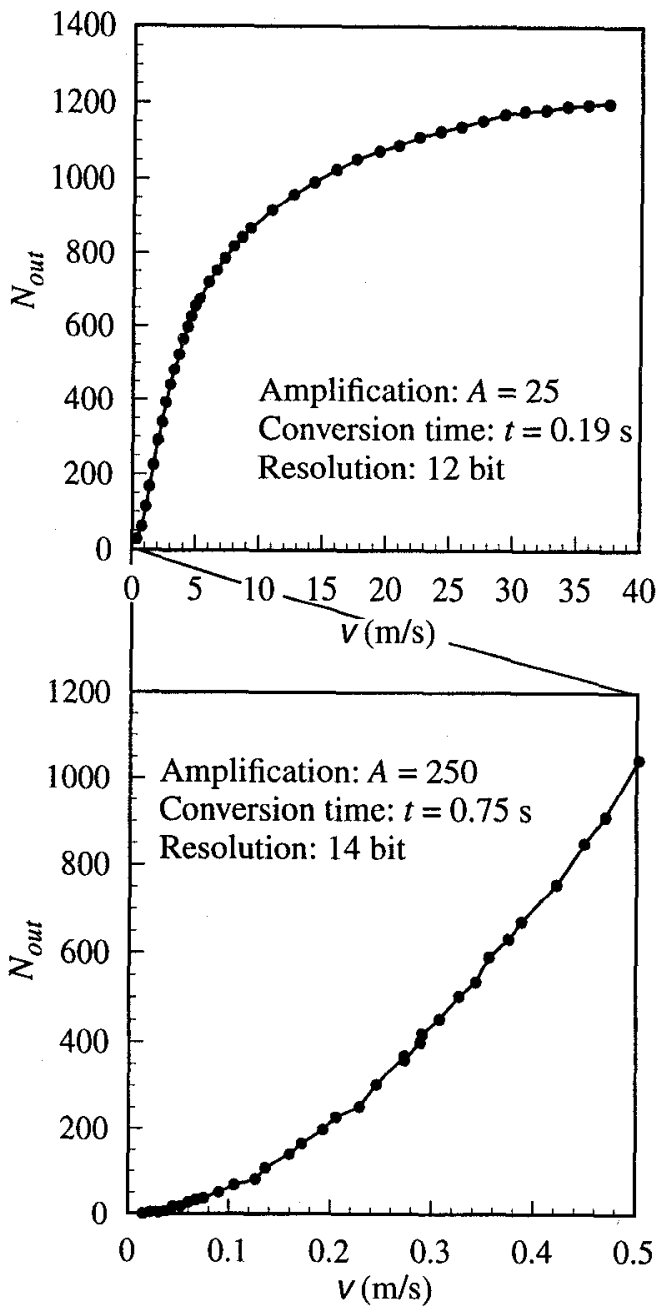

Fig. 7. Digital output signal $N_{\text {out }}$ of the anemometer in upright position as a function of air velocity $v$ for two different velocity ranges: (a) 0.5 to $38 \mathrm{~m} / \mathrm{s}$, (b) 0.02 to $0.5 \mathrm{~m} / \mathrm{s}$.

velocity range, the vertical angle dependence is below $0.5 \%$ of the output signal per degree of inclination. Temperature variations lead to changes in the sensor signal by $0.06 \% \mathrm{~K}^{-1}$. The performance parameters of the anemometer are summarized in Table 1.

\section{Conclusions}

We have reported a packaged, miniaturized anemometer microsystem. The core of the system, a thermoelectric microsensor, is fabricated using a commercial CMOS process and compatible silicon bulk micromachining. The microsystem chip contains on-chip power management, signal conditioning, and A/D conversion. The microsystem is packaged using a novel flip-chip on flex technique. An opening in the substrate allows free advection. Wind measurements are demonstrated between 0.02 and $38 \mathrm{~m} / \mathrm{s}$. The total volume of the anemometer is $2.8 \mathrm{~cm}^{3}$. The combination of bulk micromachining, commercial IC technology and flip-chip technology enabled its extreme miniaturization. 
(a)

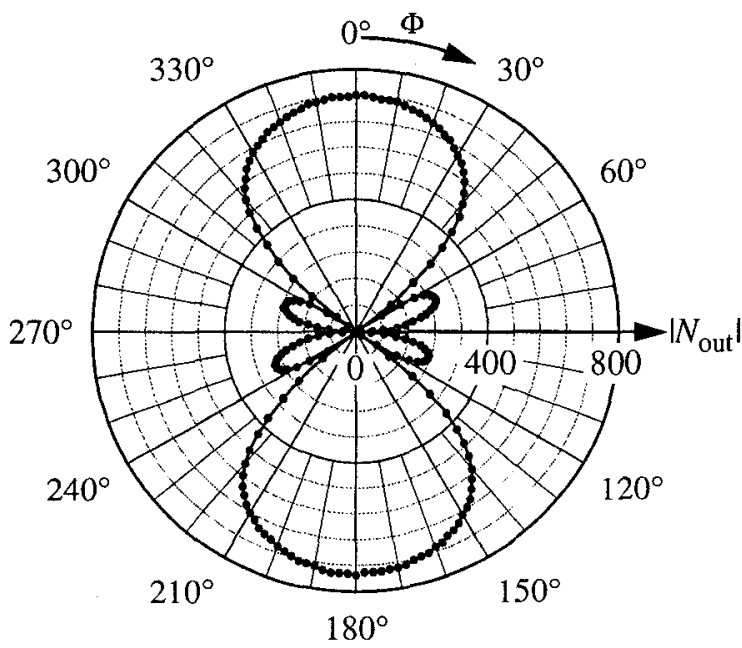

(b)

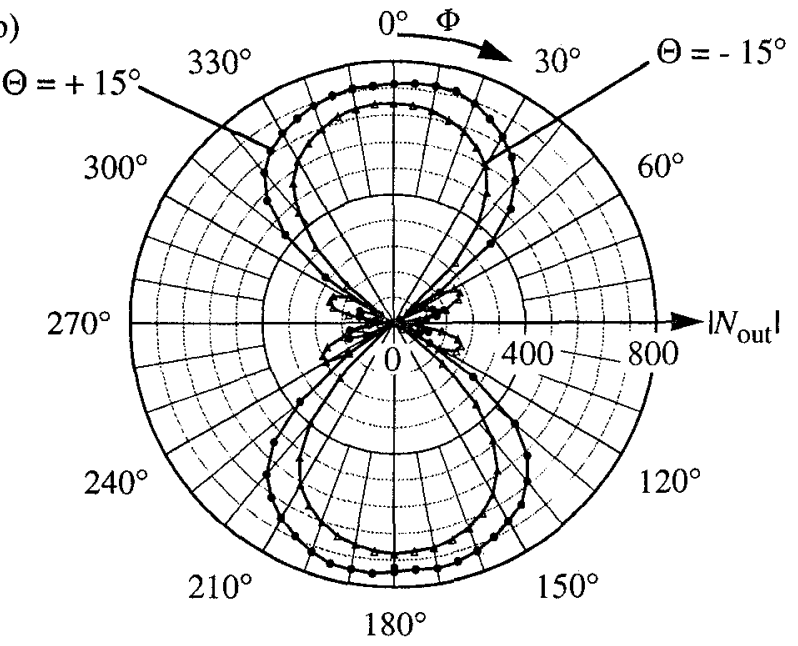

Fig. 8. Absolute value of digital output signal $N_{\text {out }}$ of the anemometer for constant air velocity $v=6 \mathrm{~m} / \mathrm{s}$ as a function of the azimuthal angle $\Phi$ of the flow channel relative to the flow direction. (a) Sensor in upright position $\left(\Theta=0^{\circ}\right)$. (b) Sensor inclined towards $\left(\Theta=+15^{\circ}\right)$ and away $\left(\Theta=-15^{\circ}\right)$ from wind direction.

Table 1: Performance of the sensor system.

\begin{tabular}{|c|c|}
\hline Feature & Value \\
\hline \hline Technology & $2 \mu \mathrm{m}$, industrial CMOS \\
\hline Packaging & Flip-chip on flex \\
\hline Power supply & $4 \mathrm{~V}$ \\
\hline Clock frequency & $22 \mathrm{kHz}$ \\
\hline Power consumption & $3 \mathrm{~mW} @ 4 \mathrm{~V}$ \\
\hline Output & digital (bitstream) \\
\hline Dynamic range & 0.02 to $38 \mathrm{~m} / \mathrm{s}(65 \mathrm{~dB})$ \\
\hline Conversion time (12 bit) & $0.19 \mathrm{~s}$ \\
\hline Conversion time (14 bit) & $0.75 \mathrm{~s}$ \\
\hline Time constant of sensor & $<0.02 \mathrm{~s}$ \\
\hline Influence of temperature & $<0.06 \% / \mathrm{K}$ \\
\hline Influence of inclination & $<0.5 \% /$ degree \\
\hline Influence of azimuth & see Fig. 8 \\
\hline
\end{tabular}

\section{Acknowledgments}

This work was supported by the Swiss Bundesamt für Bildung und Wissenschaft (BBW) through grant Nr. 93.0161 and by the Swiss Foundation for Microelectronic Research (FSRM).

\section{References}

[1] R. G. Johnson and R. E. Higashi, "A highly sensitive silicon chip microtransducer for air flow and differential pressure sensing applications", Sensors and Actuators 11, (1987) 63-72.

[2] O. Tabata, "Fast-response silicon flow sensor with an on-chip fluid temperature sensing element", IEEE Trans. Electron Devices 33, (1986) $361-365$.

[3] B. Van Oudheusen, "Integrated silicon flow sensors", PhD thesis, Technical University Delft (1989).

[4] L. Qui, E. Obermeier, A. Schubert "A microsensor with integrated heat sink and flow guide for gas flow sensing applications", Transducers '95 Digest of Technical Papers 1, (1995) 520-523.

[5] T. Neda, K. Nakamura, T. Takumi, "A polysilicon flow sensor for gas flowmeters", Transducers '95 Digest of Technical Papers 1, (1995) 548-551.

[6] M. Nagata, N. Swart, M. Stevens, A. Nathan, "Thermal based micro flow sensor optimization using coupled electrothermal numerical simulations", Transducers '95 Digest of Technical Papers 2, (1995) 447-450.

[7] E. Yoon and K. D. Wise, "An integrated mass flow sensor with on-chip CMOS interface circuitry”, IEEE Trans. Electron Devices 39, (1992) 1376-1386

[8] J. Robadey, O. Paul and H. Baltes, "Two-dimensional integrated gas flow sensors by CMOS IC technology", J. Micromech. Microeng. 5, (1995) 243-250.

[9] F. Mayer, O. Paul, and H. Baltes, "Influence of design geometry and packaging on the response of thermal CMOS flow sensors", Transducers ' 95 Digest of Technical Papers 1, (1995) 528-531.

[10] F. Mayer, G. Salis, J. Funk, O. Paul, and H. Baltes, "Scaling of thermal CMOS gas flow microsensors: experiment and simulation", Proc. IEEE Micro Electro Mechanical Systems, (IEEE 1996) 116-121.

[11] F. Mayer, M. Hintermann, H. Jacobs, O. Paul, and H. Baltes, "Thermoelectric CMOS Anemometers" Proc. SPIE Micromachined devices and Components II Vol. 2882 (SPIE 1996) 236-246.

[12] F. Mayer, O. Paul, and H. Baltes, "Flip-Chip Packaging for Thermal CMOS Anemometers", Proc. IEEE Micro Electro Mechanical Systems, (IEEE, 1997) 204-208.

[13] D. Jaeggi, "Thermal Converters by CMOS Technology", PhD thesis Nr. 11567, ETH Zurich (1996).

[14] P. Malcovati, A. Häberli, F. Mayer, O. Paul, F. Maloberti, and H. Baltes, "Combined Air Humidity and Flow CMOS Microsensor with On-Chip 15 Bit Sigma-Delta A/D Interface", VLSI Circuit Symposium '95 Digest of Technical Papers, Kyoto, Japan, (1995) 45-46.

[15] J. H. Lau, Flip chip Technologies, (McGraw-Hill, 1995).

[16] H. Baltes, O. Paul, J. G. Korvink, M. Schneider, J. Bühler, N. Schneeberger, D. Jaeggi, P. Malcovati, M. Hornung, A. Häberli, M. von Arx, F. Mayer, and J. Funk "IC MEMS Microtransducers" Proc. IEDM Micromachined devices and Components $\| I$ Vol. 2882 (IEEE 1996) 521-524.

[17] J. Robstr G. C. Temes, V. Valencic, R. Dessoulavy and Ph. Deval, "A 16-bit low voltage A/D converter", IEEE J. Solid-State Circuits, 22, (1987) $157-163$.

[18] H. Schlichting, Boundary-Layer Theory, (McGraw-Hill, 1979) 10.

[19] M. M. Zdavkovich, Flow around cirular Cylinders, (Oxford Science Publications, 1997) 70-90. 